\title{
New Protein Vector ApE1 for Targeted Delivery of Anticancer Drugs
}

\author{
N. V. Pozdniakova, ${ }^{1}$ N. V. Gorokhovets, ${ }^{1}$ N. V. Gukasova, ${ }^{1}$ \\ A. V. Bereznikova, ${ }^{2}$ and E. S. Severin ${ }^{2}$ \\ ${ }^{1}$ Centre for Convergence of Nano-, Bio-, Information and Cognitive Sciences and Technologies (Kurchatov NBIC Centre), \\ National Research Centre "Kurchatov Institute", Academician Kurchatov Square, 1, Building 348, Moscow 123182, Russia \\ ${ }^{2}$ Biological Faculty, Lomonosov Moscow State University, Vorobyovy Gory, 1, Building 12, Moscow 119234, Russia
}

Correspondence should be addressed to N. V. Pozdniakova, natpo2002@mail.ru

Received 30 November 2011; Revised 20 February 2012; Accepted 22 February 2012

Academic Editor: Kapil Mehta

Copyright ( $) 2012$ N. V. Pozdniakova et al. This is an open access article distributed under the Creative Commons Attribution License, which permits unrestricted use, distribution, and reproduction in any medium, provided the original work is properly cited.

A new chimeric gene ApE1 encoding the receptor-binding domain of the human alpha-fetoprotein fused to a sequence of 22 glutamic acid residues was constructed. A new bacterial producer strain E. coli SHExT7 ApE1 was selected for ApE1 production in a soluble state. A simplified method was developed to purify ApE1 from bacterial biomass. It was shown that the new vector protein selectively interacts with AFP receptors on the tumor cell surface and can be efficiently accumulated in tumor cells. In addition, ApE1 was shown to be stable in storage and during its chemical modification. An increased number of carboxyl groups in the molecule allows the production of cytotoxic compound conjugates with higher drug-loading capacity and enhanced tumor targeting potential.

\section{Introduction}

Active targeting of tumor cells via receptor-mediated endocytosis has the potential to specifically enhance internalization of drugs by target cells, while minimizing drug action on healthy tissues. Oncofetal proteins (alpha-fetoprotein, carcinoembryonic antigen) have been extensively used as targeted vectors for selective drug delivery, since they interact with specific surface receptors of tumor cells [1]. Human alpha-fetoprotein (AFP) comprises three domains; the third domain is responsible for binding to the cell surface of many cancer types [2]. Our previous work yielded promising results concerning the use of the recombinant third domain of human alpha-fetoprotein (rhAFP3d) as a vector in architectures for targeted drug delivery [3-5]. Small rhAFP3d peptide fragments are also currently being investigated $* *$.

A significant shortcoming of rhAFP3d and peptide fragments is that they cannot carry more than one or two covalently attached drug molecules, because the number of available reactive groups is small. To overcome this limitation, two approaches were employed; one of them involving drug-loaded rhAFP3d-conjugated nanoparticles
[5], and the other one using recombinant technologies to obtain a new rhAFP3d-based protein vector with increased number of reactive groups. The new vector protein was constructed based on the amino acid sequence of rhAFP3d with receptor-binding activity fused to a C-terminal fragment of 22 glutamic acid residues (Figure 1). Thus, the chimeric recombinant protein ApE1 was designed as a bifunctional molecule, with one part responsible for specific targeting of tumor cells and the other part planned for binding antitumor agents.

In this work, our purpose was to create the genetic construct and the producer strain for ApE1 expression, to develop an efficient method for ApE1 isolation from the biomass, and to determine the ApE1 ability to selectively interact with cancer cells.

\section{Materials and Methods}

2.1. Bacterial Strains and Plasmid. Host strains E. coli XL1Blue and E. coli SHuffle express T7 (NEB, USA) were used for cloning and expression experiments. The plasmid 


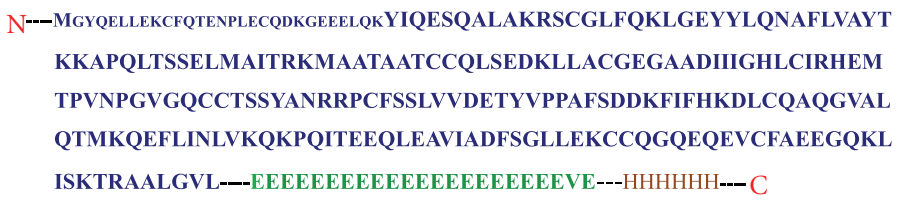

Figure 1: Amino acid composition of ApE1. Blue letters: rhAFP3d sequence, green: 22 glutamic acid residues, brown: Histag. N-, and C-terminus of the protein are shown with red letters.

pET-28a(+) (Novagene, USA) was used as a cloning and expression vector.

2.2. Plasmid Construction. Total RNA was isolated from AFPproducing human HepG2 hepatocellular carcinoma cells by the Chomczynski procedure [6]. Human AFP cDNA was obtained by reverse transcription using RTAMV reverse transcriptase and the CTTCCCCTGAAGTAAT primer complementary to the 3 ' untranslated AFP mRNA region. The DNA fragment encoding AFP amino acids 376-609 was amplified with the primers TTTTCCATGGGATACCAGGAGTTATTG (forward) and TTTTCTCGAGAACTCCCAAAGCAGCAC (reverse). The PCR product was inserted into the pET28a $(+)$ expression vector at the NcoI/XhoI site by endonuclease digestion and subsequent ligation. Following amplification in competent E. coli XL1-Blue cells, the pAFP28D3 plasmid was isolated and identified by restriction endonuclease analysis. The DNA sequence was obtained on an automated DNA sequencer. Two rare tandem arginine codons AGG AGG were substituted with CGT CGC to increase the recombinant protein yield. The polyglutamate-encoding region was synthesized by PCR using a pair of primers with complementary C-end sequences: TTCTCGAGGAAGAAGAAGAAGAAGAAGAAGAGGAGGAGGAGGAG and TTTGTCGACTTCTTCTTCTTCTTCTTCTTCСTCСТCСТCСТCСТC, and cloned into pAFP28D3 at the Xhol restriction site. The resulting plasmid pAFP28D3PolyGlu was transferred in competent E. coli XL1-Blue cells. Following amplification, plasmid DNA was isolated and identified by restriction endonuclease analysis. The presence of the chimeric apE1 gene was confirmed by DNA sequencing.

2.3. Selection of Producer Strain SHExT7 ApE1. Competent E. coli Shuffle express T7 cells were transformed with the pAFP28D3PolyGlu plasmid using the Inoue method [7]. Resulting transformant colonies were selected on a kanamycin-containing medium and tested for ApE1 expression using $1.0 \mathrm{mM} / \mathrm{L}$ of isopropyl $\beta$-D-1thiogalactopyranoside (IPTG) as an inductor. The expression level was evaluated by SDS-PAGE using the ImageJ program.

\subsection{Analysis of ApE1 Distribution between the Soluble and} Insoluble (Inclusion Bodies) Fractions. An aliquot of cell suspension in phosphate buffered saline (PBS), $\mathrm{pH} 7.8$, containing 0.3 $\mathrm{M}$ sodium chloride and protease inhibitor phenylmethylsulfonyl fluoride (PMSF) (Sigma-Aldrich, USA) was treated with $2.5 \mathrm{mg} / \mathrm{L}$ lysozyme for $15 \mathrm{~min}$ at room temperature. Then cell lysate was several times subjected to ultrasonic disintegration at $0^{\circ} \mathrm{C}$ and centrifuged at $13000 \mathrm{rpm}$ for 10 minutes at $4^{\circ} \mathrm{C}$. The supernatant and pellet were separated and analyzed by gel electrophoresis using the ImageJ program.

2.5. Isolation and Purification of ApE1 from the Soluble Fraction of SHExT7 ApE1 Producer. The soluble fraction of producer strain biomass was tested for the presence of the target protein by electrophoresis. The purification procedure included two stages. Primary purification was performed by ion exchange chromatography on DEAE-Sepharose CL6B (Sigma-Aldrich, USA). Briefly, the supernatant of the soluble fraction of producer strain biomass was loaded onto the resin preequilibrated with $\mathrm{PBS}, \mathrm{pH}$ 7.4. Next, the resin was washed with 10 volumes of $0.2 \mathrm{M}$ sodium chloride in PBS, $\mathrm{pH} 7.4$, and the target protein was eluted with $0.4 \mathrm{M}$ sodium chloride in PBS, $\mathrm{pH}$ 7.4. The protein-containing eluate was dialyzed against PBS, pH 7.4, and additionally purified by metal chelate chromatography on Chelating Sepharose $\mathrm{FF}^{\mathrm{Ni2}+}$ (Amersham, UK). Briefly, protein solution was applied onto a Chelating Sepharose $\mathrm{FF}^{\mathrm{Ni2}+}$ column equilibrated with PBS, pH 7.4, washed with $0.01 \mathrm{M}$ imidazole in PBS, pH 7.4 and then eluted with $0.25 \mathrm{M}$ imidazole in PBS, $\mathrm{pH}$ 7.4. The eluate was dialyzed twice against PBS, $\mathrm{pH} 7.4$, at $4^{\circ} \mathrm{C}$, first for $4 \mathrm{~h}$, and next for further 15 hours after changing the buffer.

\subsection{Analysis of the Target Protein ApE1}

2.6.1. SDS-PAGE. The level of ApE1 expression in the producer strain and the target protein purity at all stages of the purification procedure were analyzed by Laemmli SDSPAGE [8].

2.6.2. Protein Concentration. Protein concentration was determined by the standard procedure using a bicinchoninic acid kit (BCA) (Sigma-Aldrich, USA) with bovine serum albumin as a standard [9].

2.6.3. Detecting Free Sulfhydryl Groups. The presence of free sulfhydryl (thiol) groups per protein molecule was determined by the standard method using the Ellman's reagent $[10]$.

2.6.4. Determining the Amino Acid Composition of ApE1. For ApE1 identification, its amino acid composition was determined as described in [11]. An aliquot of the protein was subjected to acid hydrolysis, and quantitative amino acid 
analysis was performed using an amino acid analyzer (L8800, Hitachi, Tokyo, Japan).

2.7. Preparation of FITC-Labeled ApE1. FITC-labeled protein was prepared according to standard procedure. Briefly, 4 -fold molar excess of fluorescein isothiocyanate (FITC) (SigmaAldrich, USA) solution in dimethylsulfoxide was added to a protein solution in PBS, pH 7.4, and incubated in the dark for $1 \mathrm{~h}$ at room temperature, then for $18 \mathrm{~h}$ at $4^{\circ} \mathrm{C}$. FITClabeled protein was separated from free FITC on a Sephadex G-25 column (Sigma-Aldrich, USA) equilibrated with PBS, $\mathrm{pH}$ 7.4. Protein concentration was determined by BCA, and the concentration of FITC was measured by absorbance at $498 \mathrm{~nm}$.

2.8. Cell Culture. Human breast adenocarcinoma MCF-7 cells were maintained in DMEM medium (Sigma-Aldrich, USA) containing $10 \%$ fetal bovine serum (FBS, Gibco, USA) and $50 \mathrm{mg} / \mathrm{mL}$ of gentamicin in a $\mathrm{CO}_{2}$ incubator at $37^{\circ} \mathrm{C}$ in a humidified atmosphere containing $5 \% \mathrm{CO}_{2}$. Peripheral blood lymphocytes of healthy volunteers were isolated by density gradient centrifugation on a Ficoll-Paque gradient (Sigma-Aldrich, USA).

2.9. Binding and Uptake of FITC-Labeled ApE1. The binding and uptake of FITC-labeled ApE1 were studied using the technique described previously [3]. Before the experiment, cells were incubated for $2 \mathrm{~h}$ in FBS-free DMEM medium. FITC-labeled ApE1 $(0.25-4 \mu \mathrm{M})$ was added to the cell suspension in PBS and cells were incubated for $1 \mathrm{~h}$ at $4^{\circ} \mathrm{C}$ (for the binding assay) or $37^{\circ} \mathrm{C}$ (for the uptake assay). After incubation, cells were washed twice with PBS and fixed with $2 \%$ paraformaldehyde. Fluorescence was measured using an EPICS XL flow cytometer (Beckman Coulter, USA) and analyzed using the XL SYSTEM II software. The mean fluorescence intensity (MFI) was determined in each sample ( $10^{5}$ cells). The specificity of ApE1-FITC binding to the AFP receptor was determined by preliminary incubation of cells in the presence of a 30-fold excess of free ApE1 or rhAFP3d.

\section{Results and Discussion}

We obtained a plasmid construct encoding a protein with the desired amino acid sequence (Figure 1). A His-Tag-encoding sequence was also included in the construct to facilitate purification. An earlier study [12] showed that rhAFP3d was expressed in the insoluble form (as inclusion bodies) and had to be renaturated. However, renaturation was strongly complicated by the fact that the rhAFP3d contains six disulfide bonds and shows inherently hydrophobic properties. Under these considerations, E. coli SHuffle express T7 was chosen as the host strain for transformation. SHuffle is a mutant $E$. coli strain lacking two reductases (trxB and gor) and carrying an additional suppressor mutation (ahpC). SHuffle express T7 cells also contain cytoplasmic disulfide bond isomerase DsbC, which allows producing recombinant proteins with disulfide bonds in the cytoplasm.

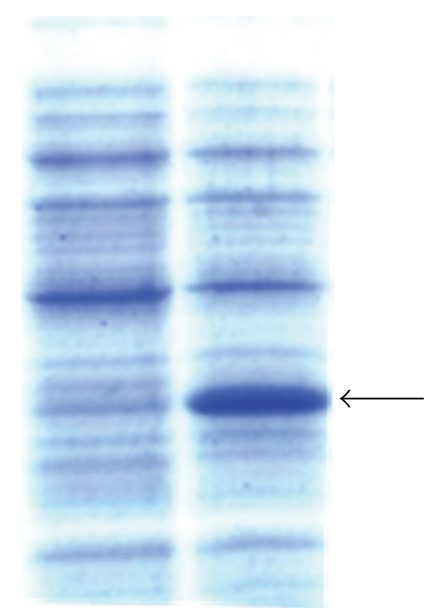

FIGURE 2: $15 \%$ SDS-PAGE of the total biomass before induction (left) and after induction (right). The arrow indicates the target protein ApE1 with predicted mass of $30 \mathrm{kDa}$.

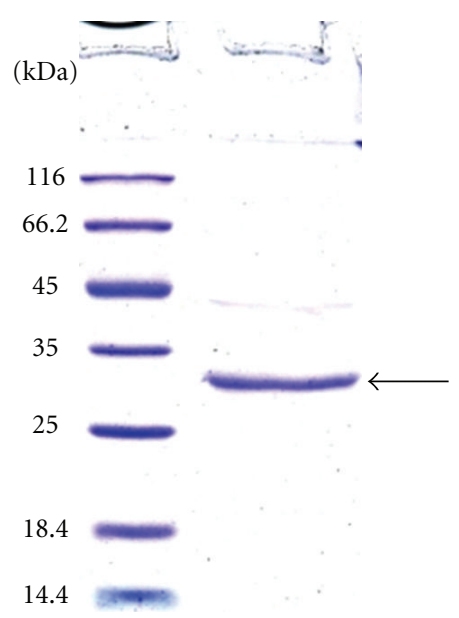

FIGURE 3: 15\% SDS-PAGE of the purified target protein. The arrow indicates the target protein ApE1 with predicted mass of $30 \mathrm{kDa}$. Molecular weight markers are shown at the left.

The observed expression level of the target protein with a predicted molecular mass of $30 \mathrm{kDa}$ was $30-40 \mathrm{mg} / \mathrm{mL}$, or $25 \%$ of total cellular proteins (Figure 2). An analysis of the ApE1 distribution between the soluble- and insoluble(inclusion bodies) protein fractions showed that more than $80 \%$ of ApE1 was synthesized in the soluble form. For this reason, we further concentrated on developing the procedure of protein isolation and purification from the soluble fraction of biomass.

Since the novel ApE1 protein is more hydrophilic than rhAFP3d and is synthesized in the soluble form, the isolation and purification procedure is not complicated by refolding. After trying out several methods, we chose the two-step purification procedure described above as the simplest and the most efficient one. The target protein yield at successive purification stages is shown in Table 1.

Target protein purified by this method (Figure 3 ) in the concentration of $1.5-2.0 \mathrm{mg} / \mathrm{mL}$ had $95 \%$ purity and showed 
TABLE 1: Yield of the target protein ApE1 in successive purification stages.

\begin{tabular}{lcc}
\hline Stages & ApE1* output (from 1 L of culture medium), mg & Yield, \% \\
\hline Biomass & 40 & 100 \\
Soluble fraction & 32 & 80 \\
1st purification step (DEAE-Sepharose CL6B) & 30 & 75 \\
2nd purification step (Chelating Sepharose FF) & 28 & 70 \\
Purified ApE1 & 28 & 70 \\
\hline
\end{tabular}

${ }^{*}$ The target protein amounts were determined using the ImageJ program after SDSPAGE.

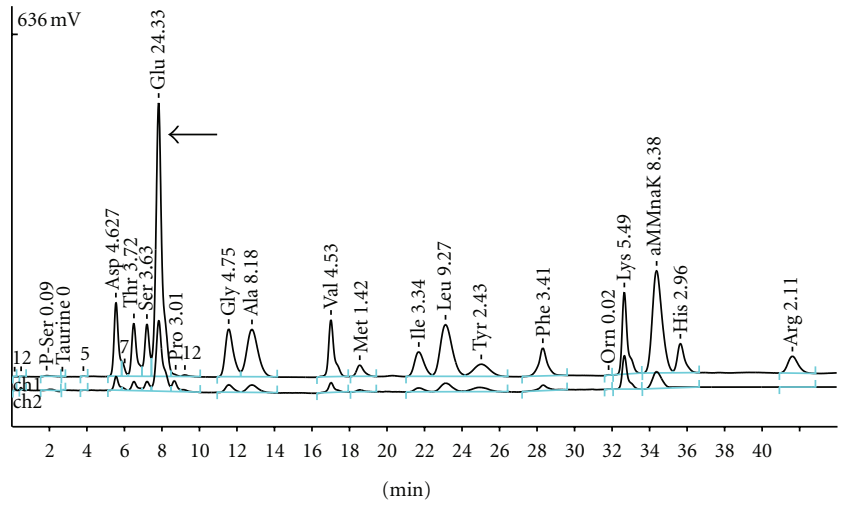

FIGURE 4: Elution profile of ApE1 hydrolysate. Arrow indicates the retention time of the glutamic acid.

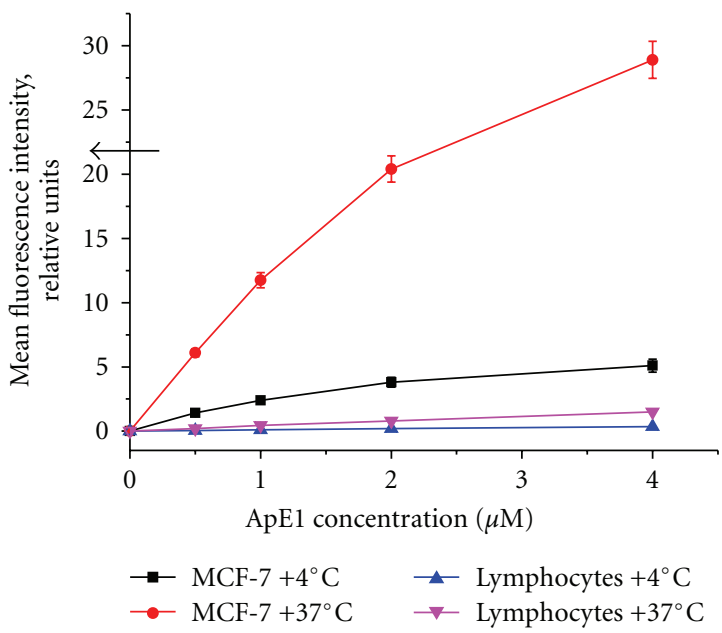

FIgUre 5: Binding and uptake of FITC-labeled ApE1 into human breast adenocarcinoma MCF-7 cells and peripheral blood lymphocytes.

lack of thiol groups in the molecule. The latter fact allows us to speculate that ApE1 refolding occurs during the growth of cell cultures.

An analysis of amino acid composition was performed to confirm the presence of 22 glutamic acid residues, and showed that the amino acid content of target protein (Figure 4) corresponded to the predicted.

To determine whether the modified protein retained the ability to bind to cancer cells and to be accumulated in them, an FITC-labeled derivative of ApE1 was synthesized. The protein/fluorescein molar ratio was defined as the ratio of moles of protein to moles of FITC in the conjugate. To obtain this ratio, ApE1 concentration was determined by BCA, and FITC concentration was determined by measuring the conjugate absorbance at $495 \mathrm{~nm}$. The protein/FITC molar ratio in FITC-labeled ApE1 was 1/1. The binding and uptake of FITC-labeled ApE1 were studied in MCF-7 human breast adenocarcinoma cells, which are characterized by a high level of AFP receptor expression (300000 receptors per cell) [2]. Flow cytometry analysis confirmed the ability of ApE1 to bind to MCF-7 cells (Figure 5). Incubation of MCF-7 cells with FITC-labeled ApE1 at $37^{\circ} \mathrm{C}$ resulted in high levels of accumulation of FITC-labeled ApE1 in the cells, indicating the possible role of receptor-mediated endocytosis in the uptake of ApE1 by tumor cells. Unstimulated peripheral blood lymphocytes were characterized with significantly lower binding and uptake of FITC-labeled ApE1 than MCF7 cells, which confirmed the specificity of ApE1 binding to tumor cells.

It was shown previously that the specificity of rhAFP3d binding to AFP receptors on MCF-7 cells was the same as that of AFP [3]. In this work, we analyzed the ability of FITC-labeled ApE1 to specifically bind to AFP receptors in the presence of excessive amounts of ApE1 or rAFP3d. The level of ApE1-FITC binding to MCF-7 cells was significantly decreased in the presence of a 30-fold excess of free ApE1 or rhAFP3d, which confirmed the specificity of ApE1 binding to the AFP receptor on the surface of tumor cells (Figure 6). Both ApE1 and rhAFP3d blocked the ApE1-FITC binding equally. Apparently, ApE1 competes with rhAFP3d for the binding site of the AFP receptor, and the receptor-binding activity of ApE1 is similar to that of rhAFP3d.

\section{Conclusion}

Previous studies have shown the efficiency of rhAFP3d as a vector in the macromolecule architectures that can selectively target cancer cells $[4,5]$. Based on the rhAFP3d sequence, we obtained a chimeric protein named ApE1, with a higher content of carboxyl groups that can be used for covalent attachment of anticancer drugs. In this work, we developed the procedure for ApE1 isolation and purification from producer biomass. The specificity of ApE1 binding to the AFP receptor and the high level of accumulation of ApE1 in tumor cells suggest that ApE1 can be efficiently used as a vector molecule for targeted delivery of anti-cancer drugs. 


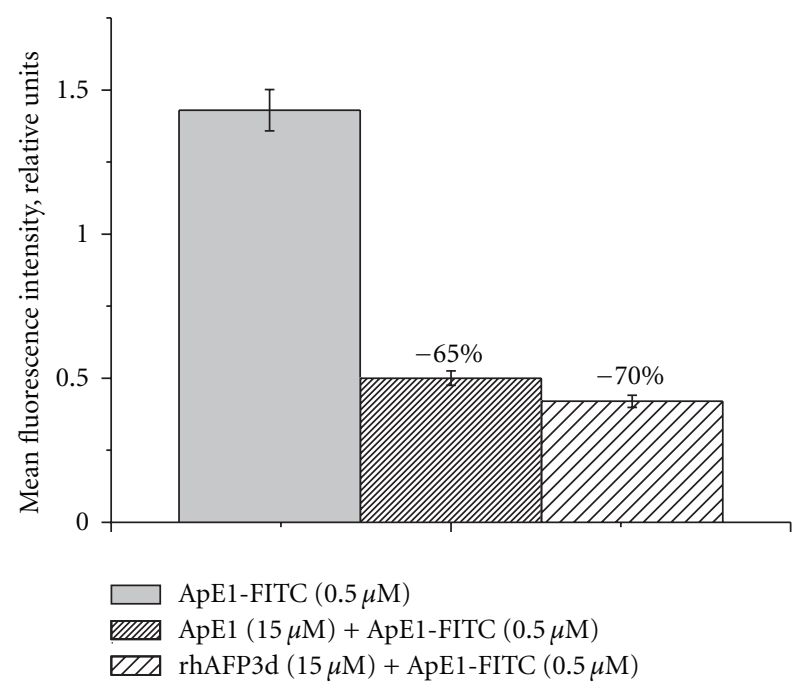

Figure 6: Inhibition of FITC-labeled ApE1 binding to MCF-7 cells in the presence of excess of free ApE1 or rhAFP3d.

\section{Acknowledgment}

This work was supported by the Russian Foundation for Basic Research, Project no. 10-04-01579-a.

\section{References}

[1] N. B. Feldman, S. M. Kiselev, N. V. Gukasova, G. A. Posypanova, S. V. Lutsenko, and S. E. Severin, "Antitumor activity of $\alpha$-fetoprotein conjugate with doxorubicin in vitro and in vivo," Biochemistry, vol. 65, no. 8, pp. 967-971, 2000.

[2] M. B. Nitsvetov, A. V. Rodina, E. Y. Moskaleva et al., "Comparison of binding rates of anti-AFPR monoclonal antibodies and AFP during their interactions with human tumor cells," Voprosy Biologicheskoi, Meditsinskoi i Farmatsevticheskoi Khimii, vol. 3, pp. 19-25, 2001.

[3] G. A. Posypanova, N. V. Gorokhovets, V. A. Makarov et al., "Recombinant alpha-fetoprotein c-terminal fragment: the new recombinant vector for targeted delivery," Journal of Drug Targeting, vol. 16, no. 4, pp. 321-328, 2008.

[4] A. V. Godovannyi, M. V. Savvateeva, and A. I. Sotnichenko, "The study of antitumor activity of conjugate recombinant $\mathrm{C}$ terminal domain of alpha-fetoprotein with cisplatin in vitro," Molecular Medicine, vol. 1, pp. 44-48, 2011.

[5] A. V. Godovannyi, E. A. Vorontsov, N. V. Gukasova et al., "Targeted delivery of paclitaxel-loaded recombinant $\alpha$-fetoprotein fragment-conjugated nanoparticles to tumor cells," Doklady Biochemistry and Biophysics, vol. 439, no. 1, pp. 158-160, 2011.

[6] P. Chomczynski and N. Sacchi, "Single-step method of RNA isolation by acid guanidinium thiocyanate-phenolchloroform extraction," Analytical Biochemistry, vol. 162, no. 1, pp. 156-159, 1987.

[7] Molecular Cloning: A Laboratory Manual, Third Edition, vol. 13, Cold Spring Harbor Laboratory Press, Cold Spring Harbor, New York, NY, USA.

[8] U. K. Laemmli, "Cleavage of structural proteins during the assembly of the head of bacteriophage T4," Nature, vol. 227, no. 5259 , pp. 680-685, 1970.
[9] P. K. Smith, R. I. Krohn, and G. T. Hermanson, "Measurement of protein using bicinchoninic acid," Analytical Biochemistry, vol. 150, no. 1, pp. 76-85, 1985.

[10] G. L. Ellman, “Tissue sulfhydryl groups," Archives of Biochemistry and Biophysics, vol. 82, no. 1, pp. 70-77, 1959.

[11] A. Tsugita and J. J. Scheffler, "A rapid method for acid hydrolysis of protein with a mixture of trifluoroacetic acid and hydrochloric acid," European Journal of Biochemistry, vol. 124, no. 3, pp. 585-588, 1982.

[12] O. A. Sharapova, N. V. Pozdniakova, D. K. Laurinavichiute et al., "Purification and characterization of recombinant human alpha-fetoprotein fragment, corresponding to the C-terminal structural domain," Bioorganicheskaia Khimiia, vol. 36, no. 6, pp. 760-768, 2010. 

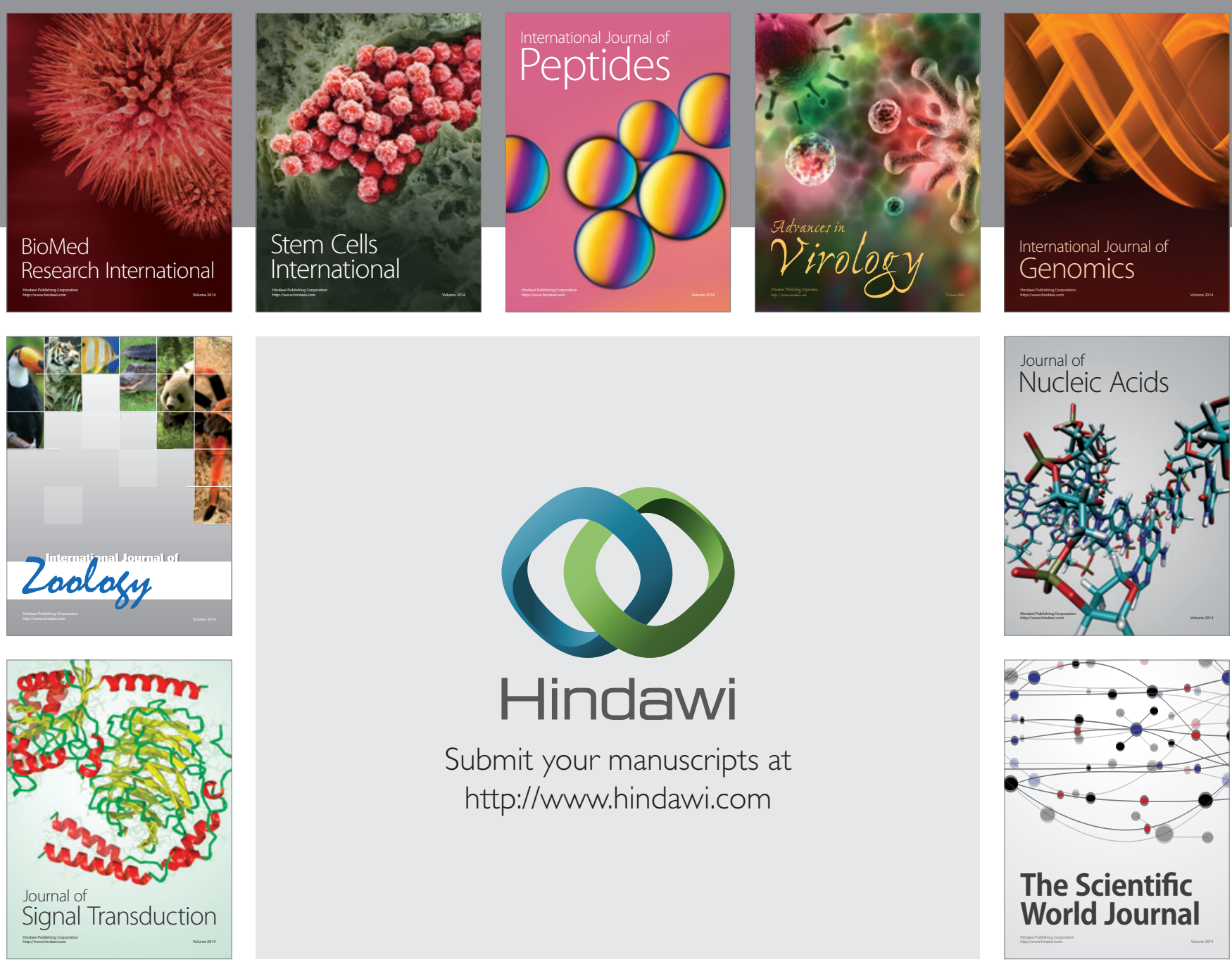

Submit your manuscripts at

http://www.hindawi.com
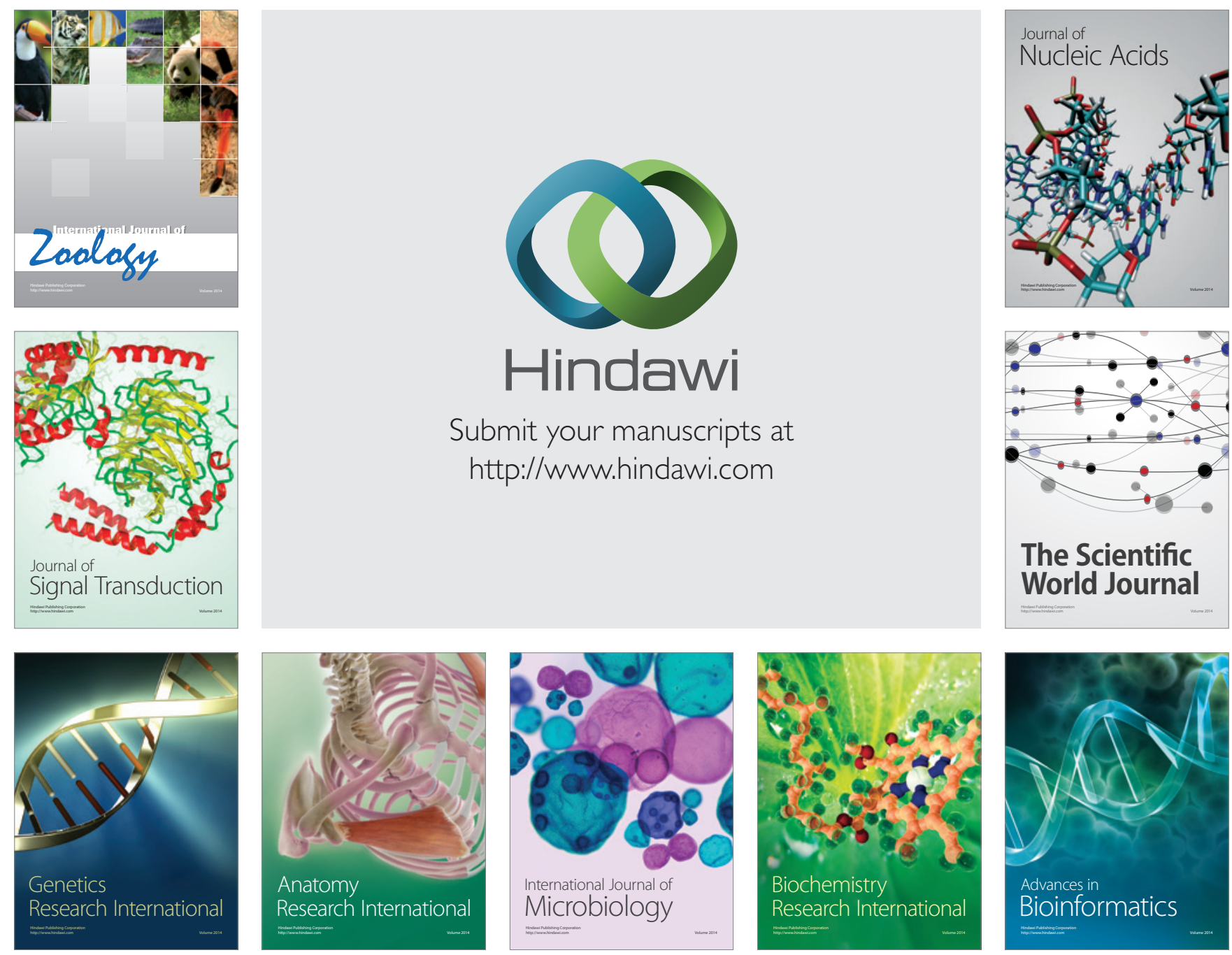

The Scientific World Journal
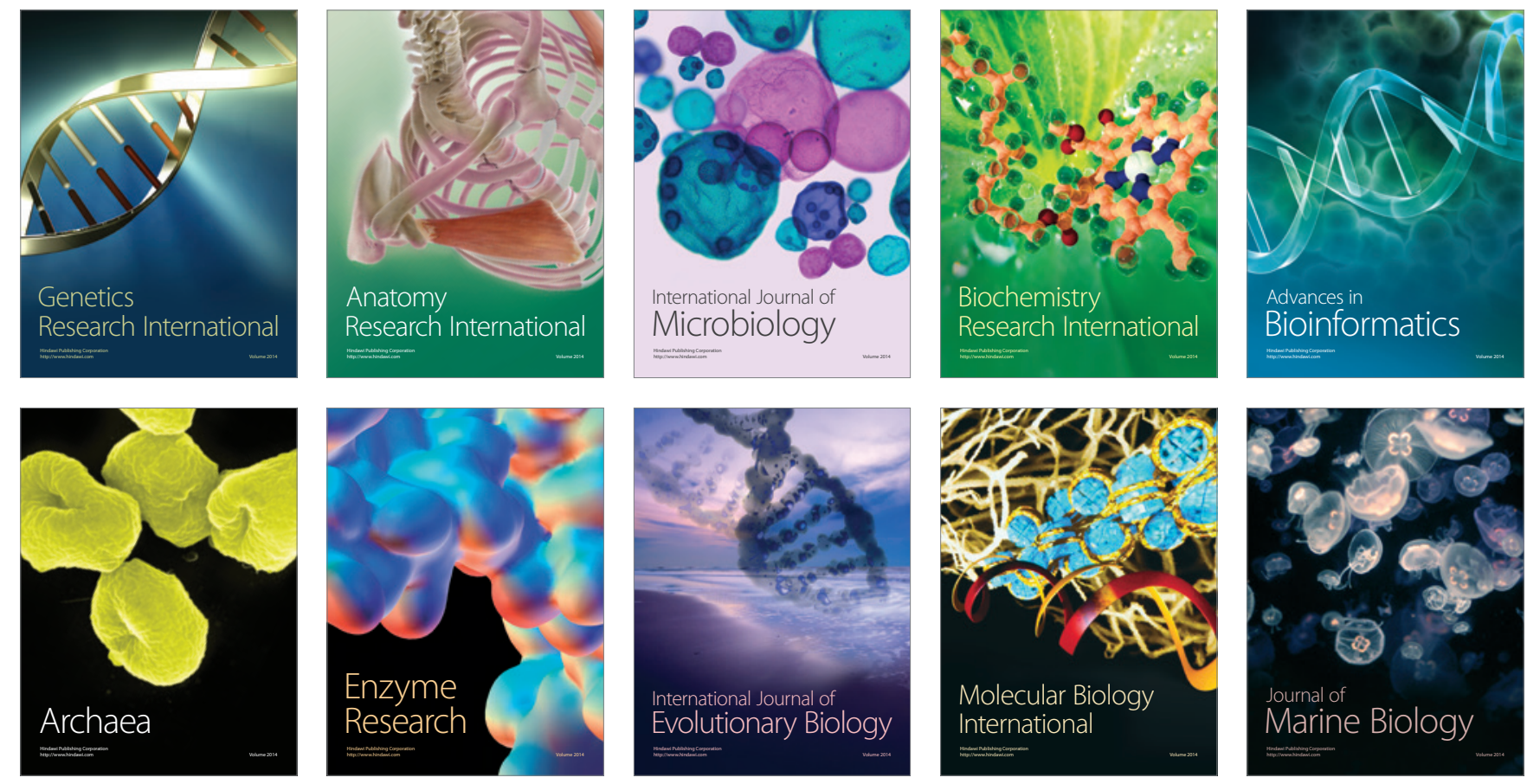